\title{
The influence of antiferroelectric compounds on helical pitch of orthoconic W-1000 mixture
}

\author{
Aleksandra Chełstowska, Michał Czerwiński, Marzena Tykarska and Noureddine Bennis
}

\begin{abstract}
The influence of six antiferroelectric compounds on the helical pitch of mixture W-1000, which was reported as long pitch orthoconic antiferroelectric liquid crystalline mixture, was checked by spectrophotometry and polarimetry methods. The electro-optical properties for the mixture with the longest pitch were measured. An improvement in electro-optical response due to the long pitch is reported. The novelty in electro-optical properties is the good symmetry response.
\end{abstract}

Keywords: liquid crystalline antiferroelectric compounds; orthoconic mixtures; helical pitch

\section{Introduction}

Orthoconic antiferroelectric liquid crystals (OAFLCs) are compounds for which molecules are tilted in layers by $45^{\circ}$. It results in unique electro-optical properties. Such materials are positive uniaxial. In comparison with conventional antiferroelectric liquid crystalline materials, they have fewer structural defects in liquid crystalline cells.[1-3]

Helical pitch is one of the fundamental parameters determining possible application of OAFLC materials. The pitch of most OAFLCs is below $1 \mu \mathrm{m}$, which makes OAFLC difficult to be stabilised by surface. It also induces asymmetry in the electro-optical response when the cell is driven with DC compensated waveform.[4] In this case, when the voltage is reduced to generate intermediate levels, the cell shows transmission variation between positive and negative cycle. This drawback results in unacceptable grey level and flickering in video rate applications.[4] Thus, the material with longer helical pitch is desirable.

The helical structure of the antiferroelectric phase may show a complicated temperature dependence. The helical pitch in this phase can increase or decrease with temperature, or increase and then decrease with a discontinuity between, exhibiting a change in helical handedness. [5-7] The usual method of changing the material parameters of liquid crystal mixtures is addition of proper dopants. Helical pitch and helical twist sense are quite sensitive to doping. [8]

Recently long pitch OAFLC mixture W-1000 was reported.[9] The aim of this work is further increase of its helical pitch as well as an improvement of its electro-optical properties. Thus, the influence of six antiferroelectric compounds on the helical pitch and helical twist sense of mixture W-1000 was checked and the electro-optical properties for the mixture with the longest pitch were measured.

\section{Experimental details}

The composition of the bicomponent eutectic mixture (W-1000) [10] exhibiting the orthoconic antiferroelectric phase is shown in Table 1.

$\mathrm{W}-1000$ is a mixture of two compounds with a phenyl biphenylate core and a semiperfluoroalkyl terminal chain ( 1 and 2 ), but differing in their oligomethylene spacer length, respectively 7 and 5 , and in the position of the fluorine substituent in the rigid core (position 2 or 3 , respectively).

The phase transitions shown by $\mathrm{W}-1000$ are as follows:

$$
\mathrm{SmC}_{\mathrm{A}} * 99.2 \mathrm{SmC} * 101.5 \mathrm{SmA} 103.5 \text { Iso }
$$

Dopants, with the structure and phase transitions presented in Table 2, were added to mixture W-1000 in the amount 20,50 and $80 \mathrm{wt} \%$. They belong to the same family of compounds as components of the host mixture.

The synthesis of compounds used for formulation of mixture W-1000 and used as dopants (A-F) has been described in Refs $[11,12]$. The phase transition temperatures, helical pitch and helical twist sense of mixture W-1000 doped with compounds A-F were measured.

Phase transitions and phase sequences were observed using a polarising microscope (BiolarPZO) equipped with heating stage (Linkam THMS 
Table 1. Composition of the eutectic mixture W-1000.

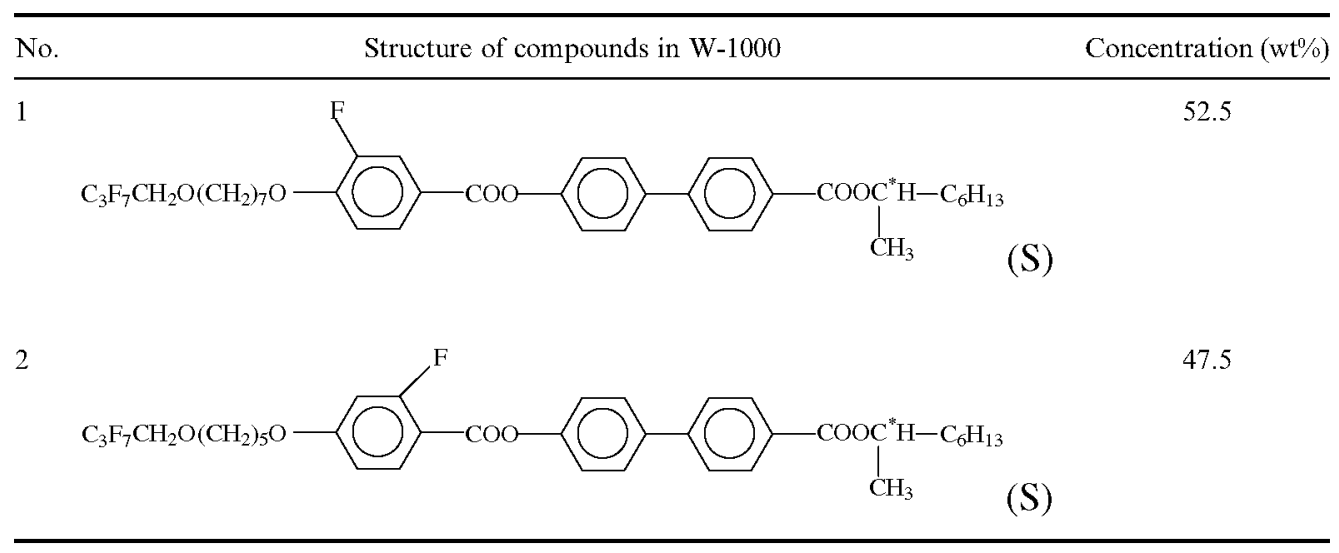

Table 2. Structure and phase transitions of antiferroelectric compounds added to mixture W-1000.

Structure of compounds

B

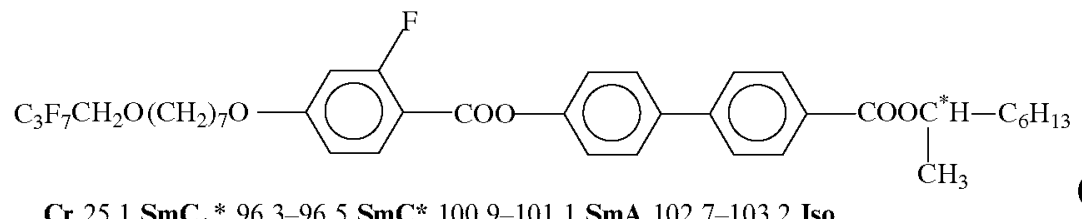

Cr 25.1 $\mathrm{SmC}_{\mathrm{A}} * 96.3-96.5 \mathrm{SmC}^{*} 100.9-101.1 \mathrm{SmA} 102.7-103.2$ Iso

$\mathrm{C}$

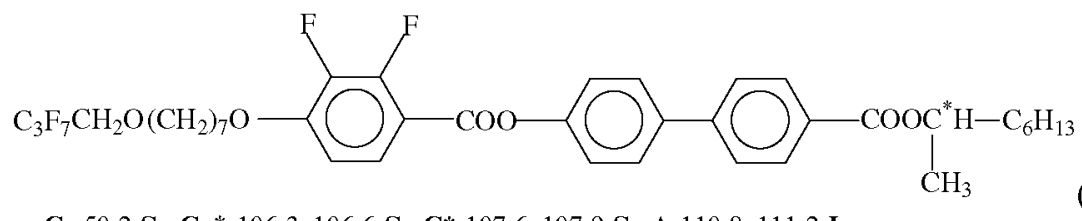

Cr 50.2 $\mathrm{SmC}_{\mathrm{A}} * 106.3-106.6 \mathrm{SmC}^{*} 107.6-107.9 \mathrm{SmA} 110.8-111.2$ Iso

D

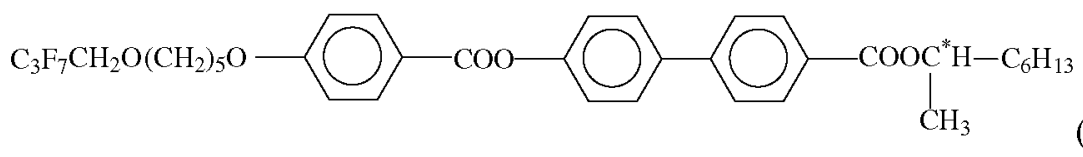

Cr $68.0 \mathrm{SmC}_{\mathrm{A}} * 123.1-123.6 \mathrm{SmC}^{*} 125.9-126.3$ Iso

E

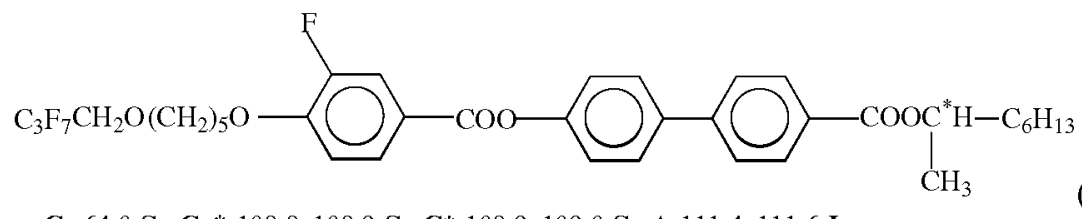

Cr $64.0 \mathrm{SmC}_{\mathrm{A}}^{*} 108.8-108.9 \mathrm{SmC}^{*} 108.9-109.0 \mathrm{SmA} 111.4-111.6$ Iso

F

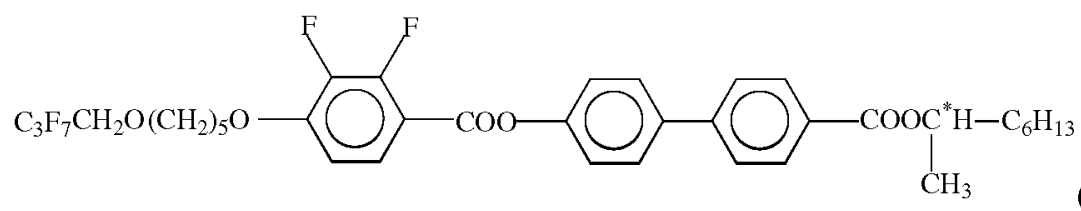

Cr $53.5 \mathrm{SmC}_{\mathrm{A}} * 109.7-110.0 \mathrm{SmC}^{*}$ 110.2-110.4 SmA $111.5-111.8$ Iso 
600 ) and temperature controller (Linkam T95-STD). Observations were performed over a heating and cooling cycle close to the phase transition region at a rate of $1^{\circ} \mathrm{C} \mathrm{min}^{-1}$. All the phases were easy to recognise from the microscopic textures without recourse to other identification methods.

The helical pitch measurements were based on selective light reflection. The measurement of light transmission was carried out using a Shimadzu UVVIS-NIR spectrometer in the range $360-3000 \mathrm{~nm}$. The tested compounds were placed on a glass plate covered with a surfactant (cetyl trimethylammonium bromide) to ensure homeotropic alignment. This orientation geometry is more favourable for correct pitch measurements. In the case of other orientation geometry, the stronger influence of surface interactions on the helical structure is observed, which in turns causes larger measurement error. The accuracy of alignment was checked by observation of texture in the SmA phase, a dark texture indicating homeotropic orientation. For the calculation of $p$ from the selective reflection band position, we used the form $p=\lambda_{\mathrm{S}} / n_{\mathrm{av}}$ for $\mathrm{SmC}_{\mathrm{A}}$ * assuming that in the spectral range of interest the average refraction index $n_{\mathrm{av}}$ is independent on temperature and wavelength (the value $n_{\mathrm{av}}=1.5$ was taken for calculation according to Ref. [13]).

AML WU7 temperature controller with Peltier element was used for controlling the temperature within the range $2-110^{\circ} \mathrm{C}$ to an accuracy of $0.1^{\circ} \mathrm{C}$. The results presented were obtained during the cooling cycle. The helical twist sense for the chiral phases was determined by the polarimetry method.[14] The temperature of the helical twist sense inversion was established by the analysis of transmitted light versus temperature of a homeotropically aligned sample under a polarising optical microscope; the brightest texture indicates an unwound structure.[5]

The cell for electro-optical measurements was 1.5 $\mu \mathrm{m}$ thick with homogeneous alignment. This surface conditioning was achieved by spin coating Nylon 6 on the indium tin oxide-coated glass, followed by curing and rubbing steps. The quasi static electro-optical response was measured by applying triangular waveform of $0.1 \mathrm{~Hz}$. Dynamic characterisation was performed employing symmetric seven levels waveform for passive multiplexing.[15] The greyscale was developed along the dynamic range employing the passive multiplexing waveform.

\section{Results and discussion}

\subsection{Phase diagrams}

Phase diagrams of systems: W-1000 mixed with compound $\mathbf{A}-\mathbf{F}$ are illustrated in Figure 1. In all cases, across the entire range of concentrations, there are three mesomorphic phases: $\mathrm{SmC}_{\mathrm{A}}{ }^{*}, \mathrm{SmC}^{*}$ and $\mathrm{SmA}$, and that the liquid crystal phase is mixed in an unlimited way. For all systems, the crystalline phase exists above room temperature only for higher concentration of dopants (above $80 \mathrm{wt} \%$ ).

\subsection{Helical parameters}

The temperature dependence of helical pitch of tested mixtures is presented in Figure 2. The sense of the helical twist ('+' - right-handed, '-' - left-handed) and the temperature of helical sense inversion (arrows) are illustrated.

Temperature dependence of the helical pitch is not the same in all cases. Addition of a three ring ester with seven methylene groups $\left(-\mathrm{CH}_{2}-\right.$ ) (dopant $\left.\mathbf{A}\right)$ to a mixture W-1000 causes an increase of the helical pitch of left-handed helix at higher temperature and slight increase of the helical pitch for right-handed helical structure.

Introducing to the core one fluorine atom (dopant B) changes the dependence of helical pitch upon temperature. At high temperatures, the helical pitch of left-handed structure decreases. At low temperatures, helical pitch increases significantly.

Adding dopant $\mathbf{C}$ to the mixture $\mathrm{W}-1000$ causes a mild decrease of the helical pitch at higher temperatures and left-handed helix. At low temperatures, we observed an increase of helical pitch and right-handed helical structure.

Reducing terminal chain to five methylene groups $\mathrm{CH}_{2-}$ (dopant D) introduces major changes in the dependence of the helical pitch upon temperature. At low temperatures, the helical pitch is significantly reduced. Helical structure is right-handed. At high temperatures, the helical pitch increases, and for the values of $50 \%$ and $80 \%$ dopant $\mathrm{D}$ as well as for pure dopant $\mathbf{D}$ helical pitch values are beyond the measuring range.

Adding to the mixture W-1000 dopant $\mathbf{E}$ and dopant $\mathbf{F}$ does not introduce substantial changes in the dependence of the helical pitch upon temperature in relation to the dopant $\mathbf{D}$. At low temperatures, the helical pitch is reduced, and at the high temperature the helical pitch is increased.

Summary dependence of helical pitch upon concentration is shown in Figure 3 for the low (a) and high (b) temperatures. At low temperatures, the longer terminal chain compounds cause a small (A) or significant (C) increase in the helical pitch upon concentration of added dopant. Reducing the length of terminal chain causes a decrease of the helical pitch upon concentration in all three cases $(\mathbf{D}, \mathbf{E}$ and $\mathbf{F})$. At high temperatures, the dependence of the helical pitch 
(a)

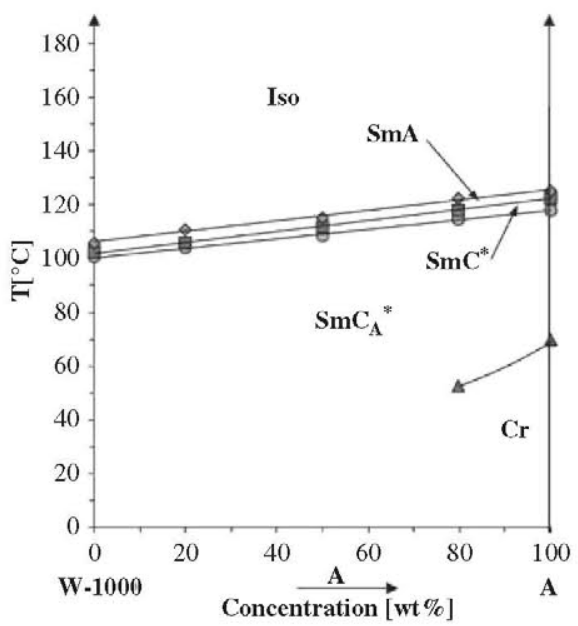

(c)

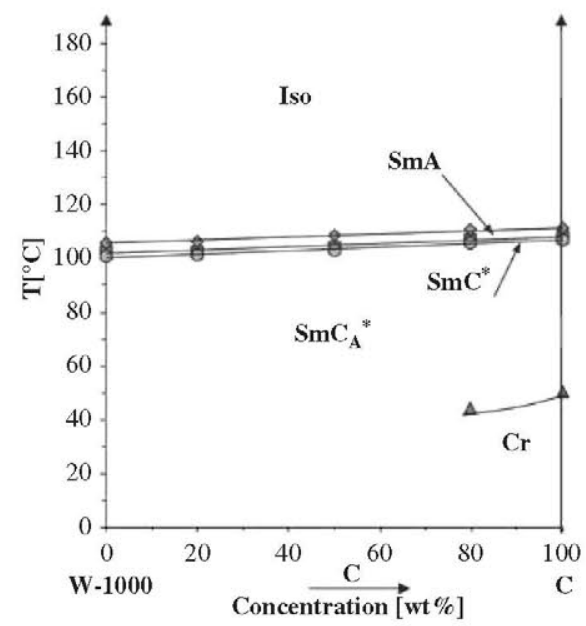

(e)

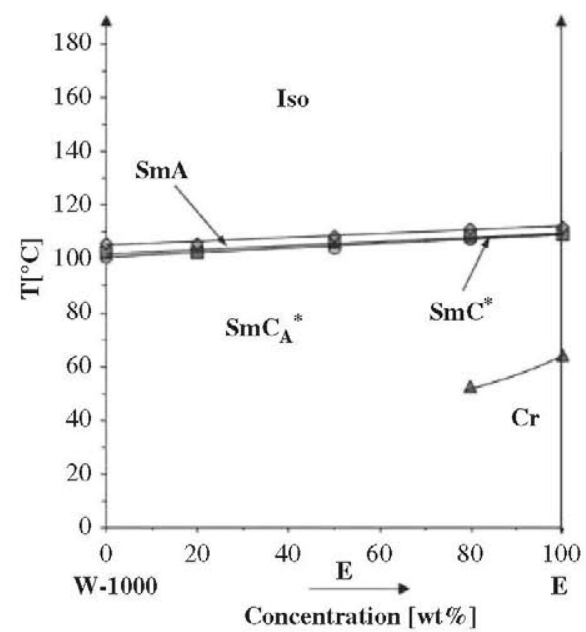

(b)

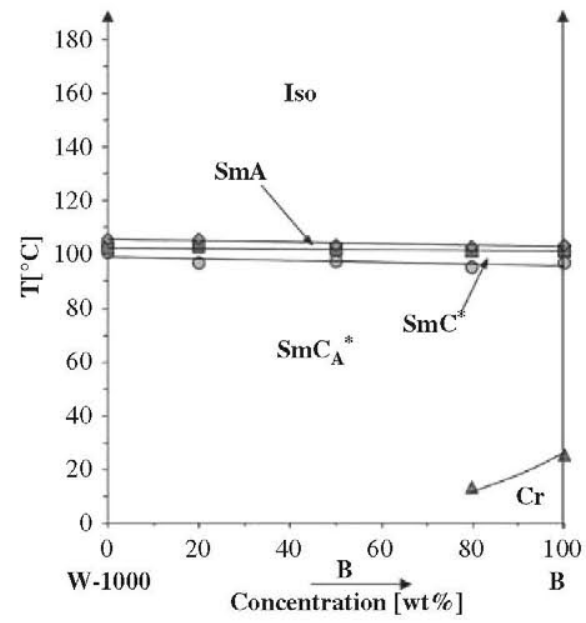

(d)

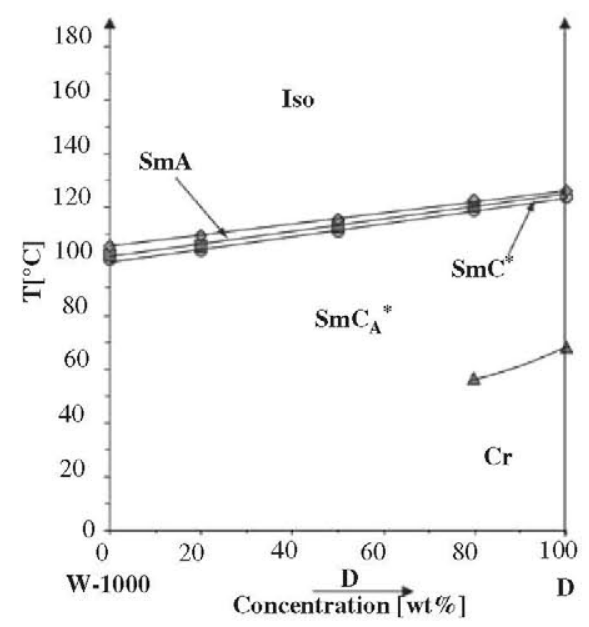

(f)

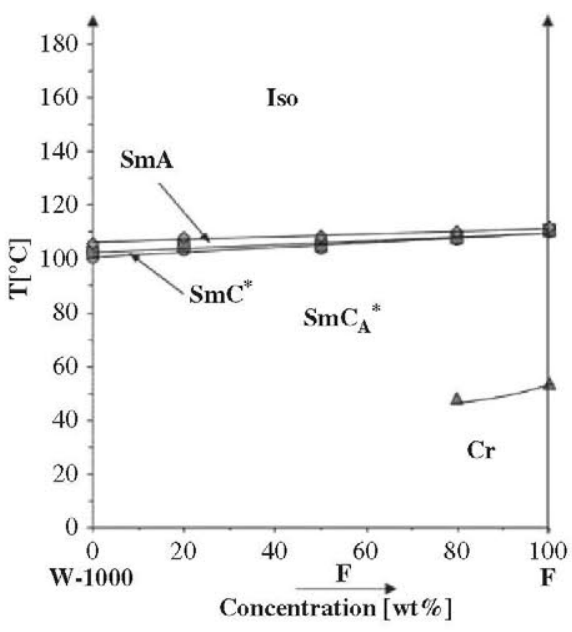

Figure 1. The phase diagrams of W-1000 mixed with compounds: $\mathbf{A}$ (a), $\mathbf{B}$ (b), $\mathbf{C}$ (c), $\mathbf{D}$ (d), $\mathbf{E}$ (e) and $\mathbf{F}$ (f). 
(a)

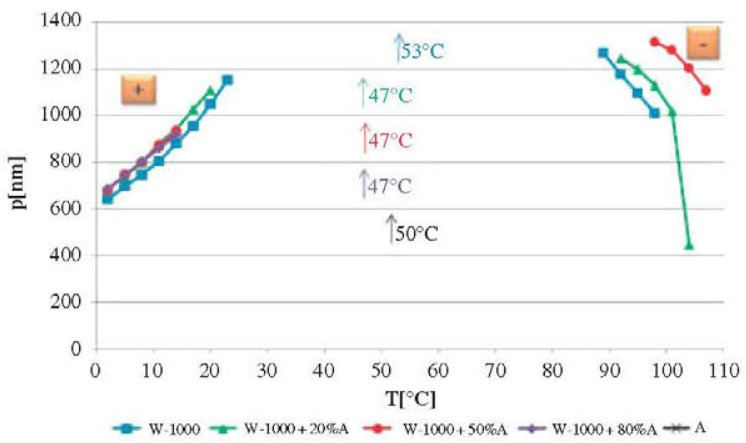

(c)

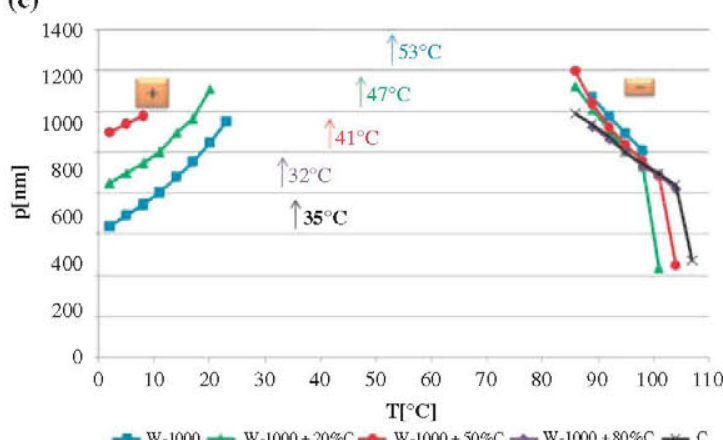

(e)

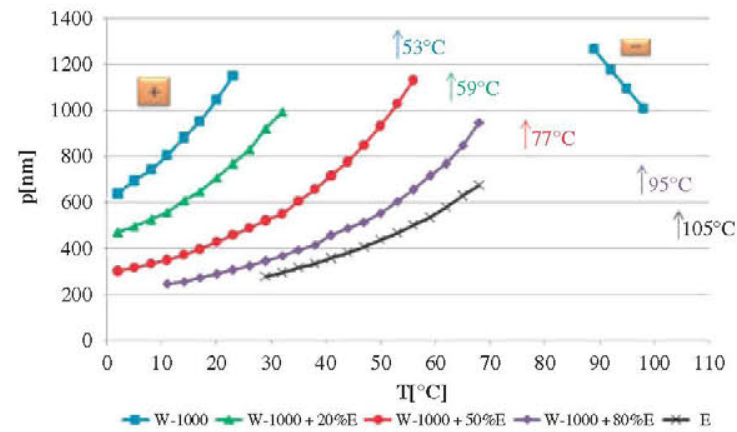

(b)

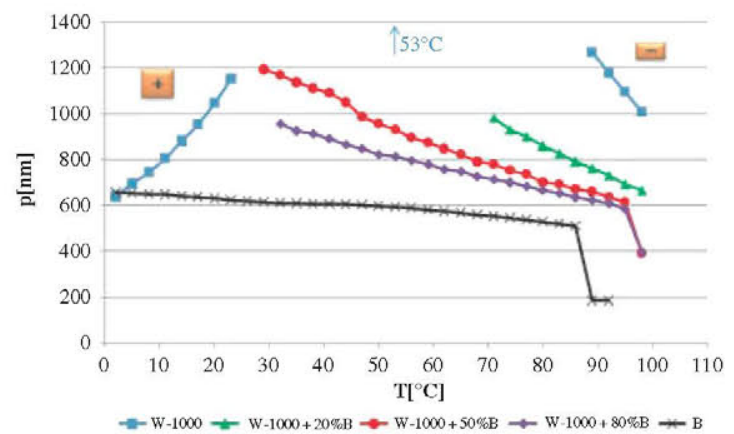

(d)

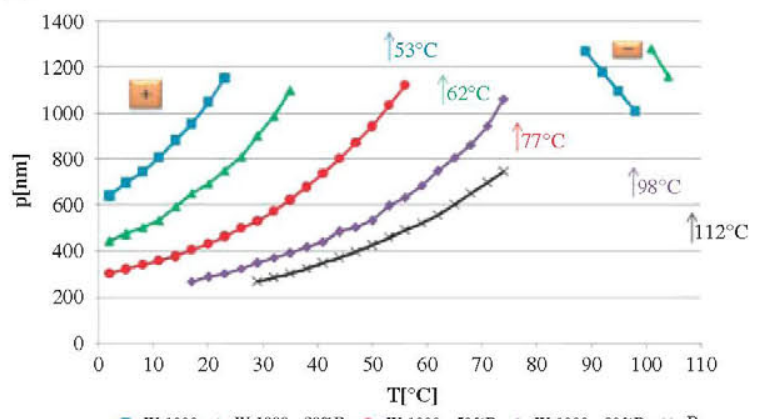

(f)

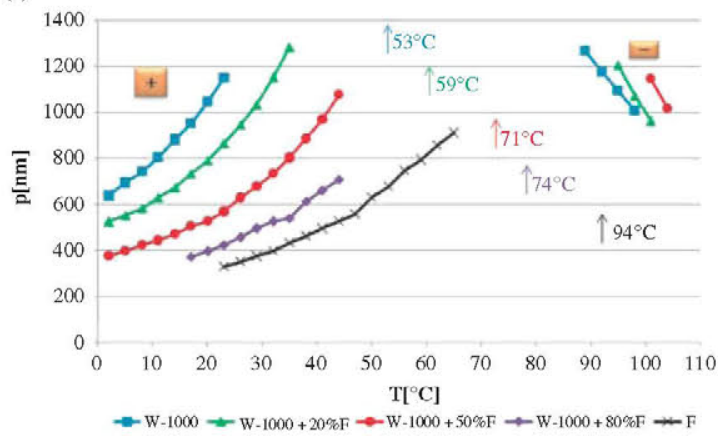

Figure 2. (colour online) Dependence of helical pitch upon temperature for mixture W-1000 doped with: A (a), B (b), C (c), D (d), $\mathbf{E}$ (e) and $\mathbf{F}$ (f). Arrows indicate the temperature at which the helix is unwound.

upon concentration is not unequivocal. In the case of compounds of the chain with seven carbon atoms in oligomethylene spacer the helical pitch increases (A), decreases (B) or stays without changes (C) upon concentration. Reducing the terminal chain to five carbon atoms in oligomethylene spacer causes the increase of the helical pitch upon concentration $(\mathbf{F})$.

Temperature, referred to phase transition $\mathrm{SmC}_{\mathrm{A}}{ }_{-}$ $\mathrm{SmC}^{*}$, of helical twist sense inversion versus concentration of dopants is shown in Figure 4.

Compounds with seven carbon atoms in oligomethylene spacer in the terminal chain (A, B and $\mathbf{C})$ are characterised by decreasing temperature of helical twist sense inversion upon concentrations of dopants. Reducing the terminal chain to five carbon atoms in oligomethylene spacer (D, E and F) causes the increase of the temperature of helical twist sense inversion upon concentration of added compounds. As one can see the temperature of helical twist sense inversion can easily be controlled by various dopants and their concentration.

The proposed composition of the mixture with a greater helical pitch than the mixture W-1000 is presented in Table 3 and is indicated with acronym W-304.

The phase transitions shown by W-304 are as follows: 
(a)

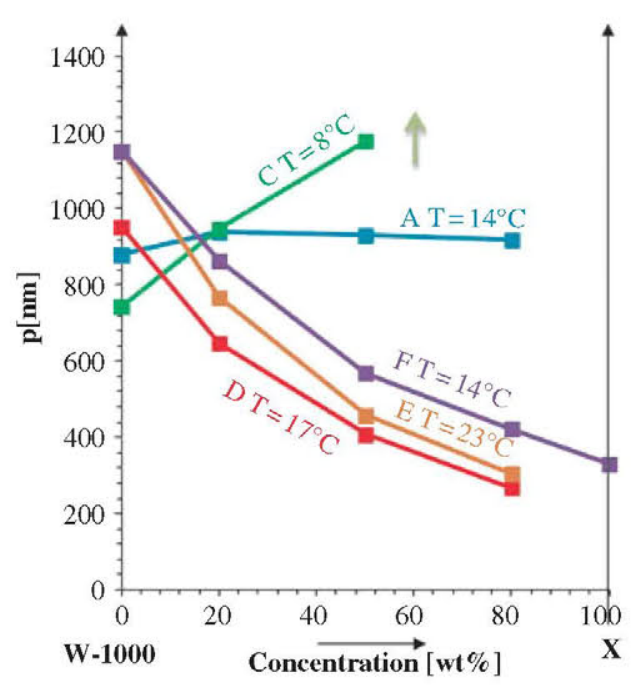

(b)

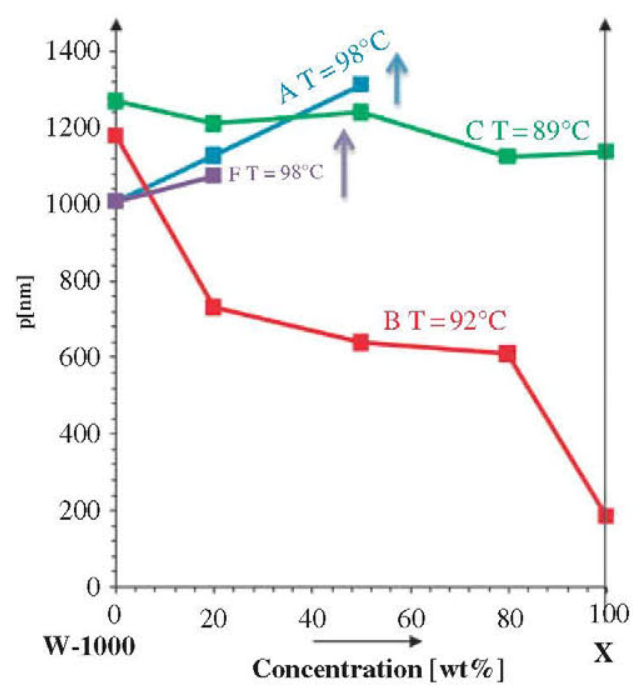

Figure 3. (colour online) Dependence of the helical pitch upon the concentration for the low (a) and high (b) temperatures.

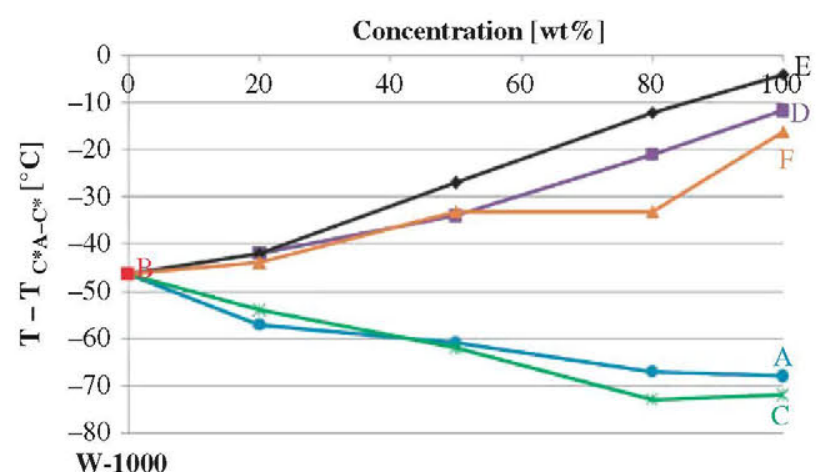

Figure 4. (colour online) Temperature, referred to phase transition $\mathrm{SmC}_{\mathrm{A}}{ }^{*}-\mathrm{SmC}^{*}$, of helical twist sense inversion versus concentration of dopants.

$$
\mathrm{Cr}<0 \mathrm{SmC}_{\mathrm{A}} * 101.6 \mathrm{SmC} * 103 \mathrm{SmA} 106 \text { Iso }
$$

\subsection{Properties of $W-304$ mixture}

As it can easily be seen from Figure 5, the optical tilt angle for mixture W-304 shows saturation below $60^{\circ}$ $\mathrm{C}$ and in these temperatures it achieves value between $43^{\circ}$ and $44^{\circ}$. It is worth pointing out that the optical tilt angle for mixture W-304 is the same than for mixture W-1000.[9]

The quasi static electro-optical response of surface stabilised OAFLC mixture W-304, measured by applying triangular waveform of $0.1 \mathrm{~Hz}$, is presented in Figure 6.
The sample was oriented between crossed polarisers in order to achieve symmetric response between hysteresis loops upon saturation. When the voltage was reduced, the symmetry was kept upon intermediate grey scale. This symmetry of the hysteresis curve is a consequence of the long pitch of the mixture which allows good alignment of the molecules. The welldefined hysteresis obtained in Figure 6 allows the optimum design of waveform for passive multiplexed waveform (Figure 7a). The results obtained for a mixture W-304 shows better electro-optical behaviour than for a mixture W-1000 reported in Ref. [9]. The mixture W-1000 shows asymmetry in hysteresis curve and higher switching voltage. However, the W-304 shows fully symmetric electro-optics with reduced switching voltage up to $4 \mathrm{~V}$ in comparison to a mixture W-1000.

This symmetry in electro-optical response is crucial to allow the pixel to be completely blanked between consecutive frames when the passive multiplexing driving is used. Consequently the transmission of a pixel in a given frame will not depend on its transmission in the previous frame avoiding flickering in video rate applications.[4] In both cases, the measured dynamic and quasi static contrast ratio are higher than 300 . The good contrast is consequence of the good alignment of the molecules and orthoconic antiferroelectric structure. When these two conditions are fulfilled, the total suppression of the pretransitional effect is achieved and the contrast is enhanced. Figure 8 shows the observed textures of rubbed cell filled with mixture W-304 
Table 3. Composition of the mixture W-304.

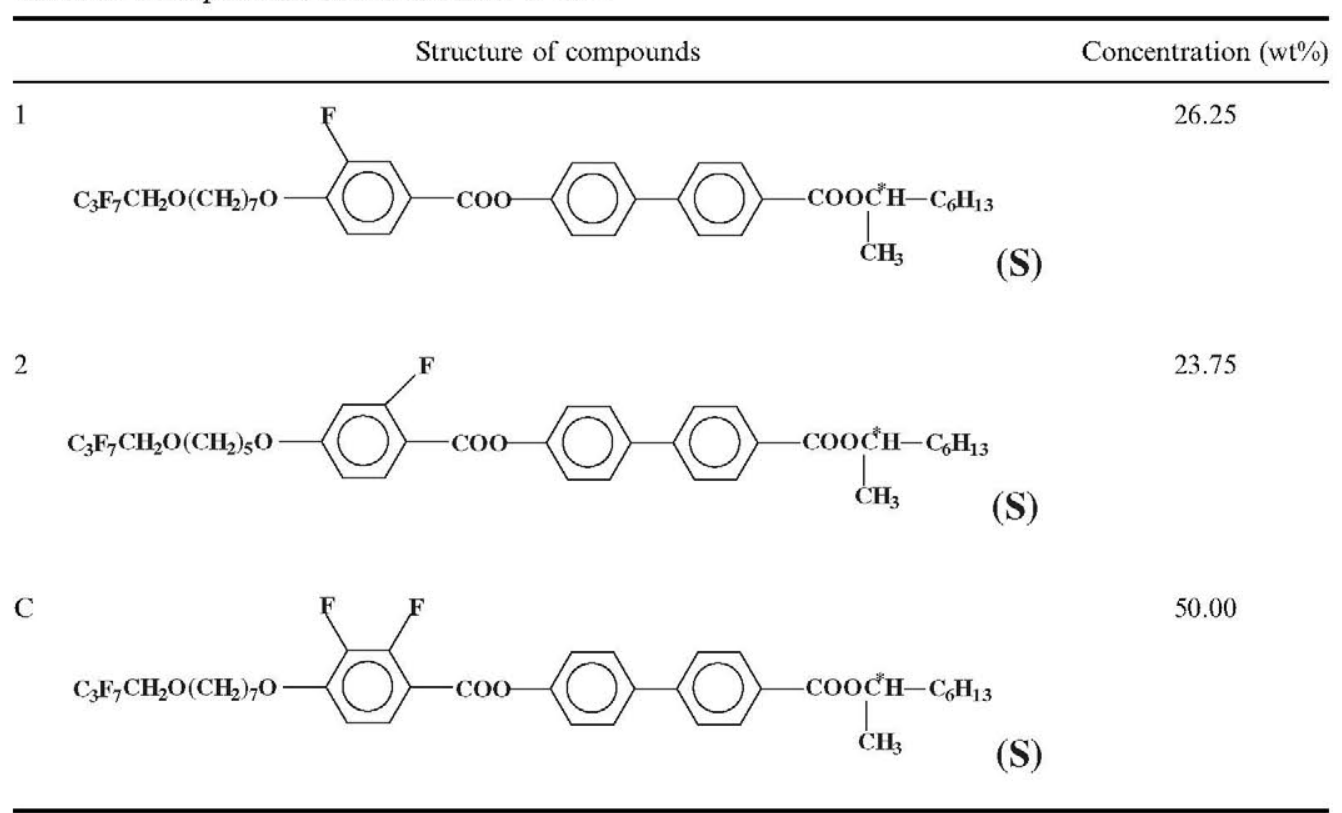

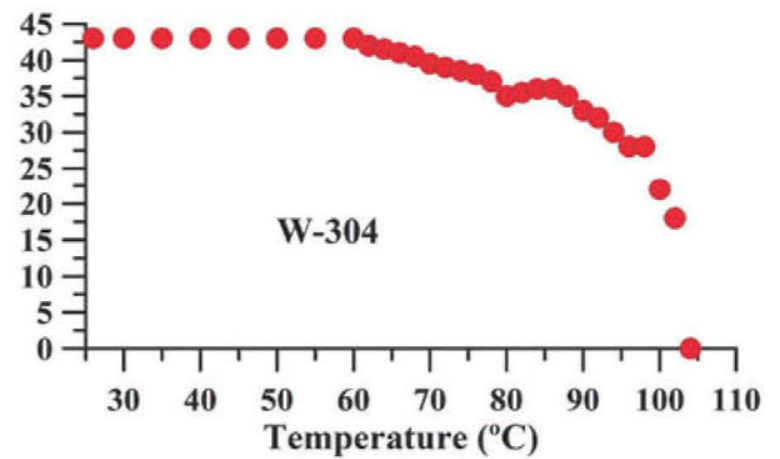

Figure 5. (colour online) The tilt angle as a function of temperature plotted for mixture W-304.

between crossed polarised when the cell is in the OFF and ON state.

\section{Conclusions}

On the basis of this study, it can be determined which of the dopants added to a mixture W-1000 cause the increase in its helical pitch.

The most preferred dopant is a three ring ester compound containing seven carbon atoms in oligomethylene spacer of the terminal chain and having a laterally substituted fluorine atoms in the core (C) results in a significant increase in helical pitch at low temperatures, which results in mixtures desired for application.

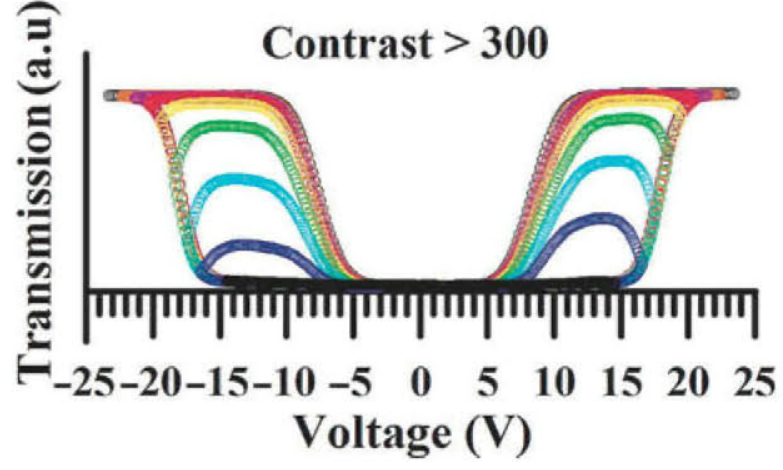

Figure 6. (colour online) Electrooptical response of OAFLC mixture W-304 to $0.1 \mathrm{~Hz}$ triangular AC signals with different amplitudes.

A dopant, which can also be used in mixtures with long helical pitch, is a compound having seven carbon atoms in oligomethylene spacer of terminal chain and does not contain laterally substituted fluorine atoms (A) - it causes a slight increase in helical pitch at low temperatures.

All compounds containing five carbon atoms in oligomethylene spacer of terminal chain $(\mathbf{D}, \mathbf{E}$ and $\mathbf{F})$ as well as the compound containing seven carbon atoms in oligomethylene spacer and one fluorine atom substituted to the core $(3 \mathrm{~F} 7 \mathrm{HPhF})$ cause twisting of the helix over a temperature range and should not be added to a mixture W- 1000 . 

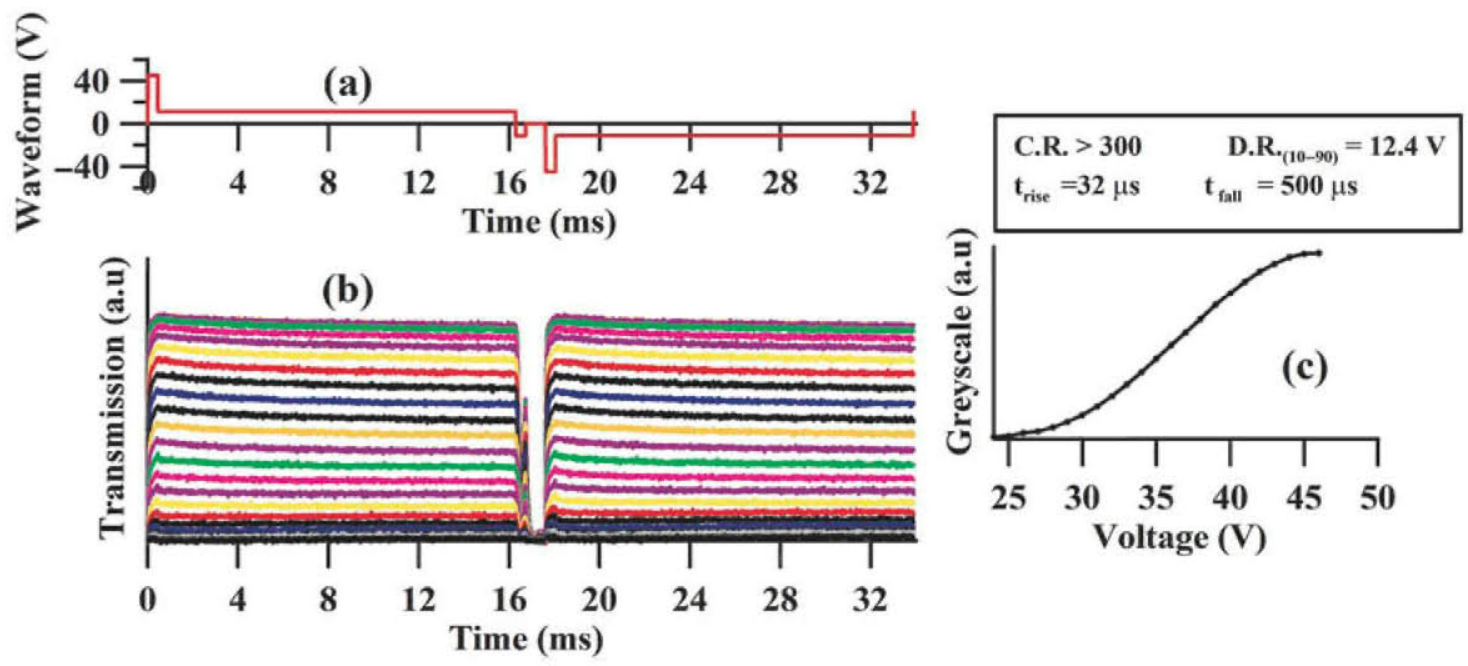

Figure 7. (colour online) Characterisation sheet of W-304: (a) seven levels waveform, (b) electro-optical transmission levels and (c) greyscale.

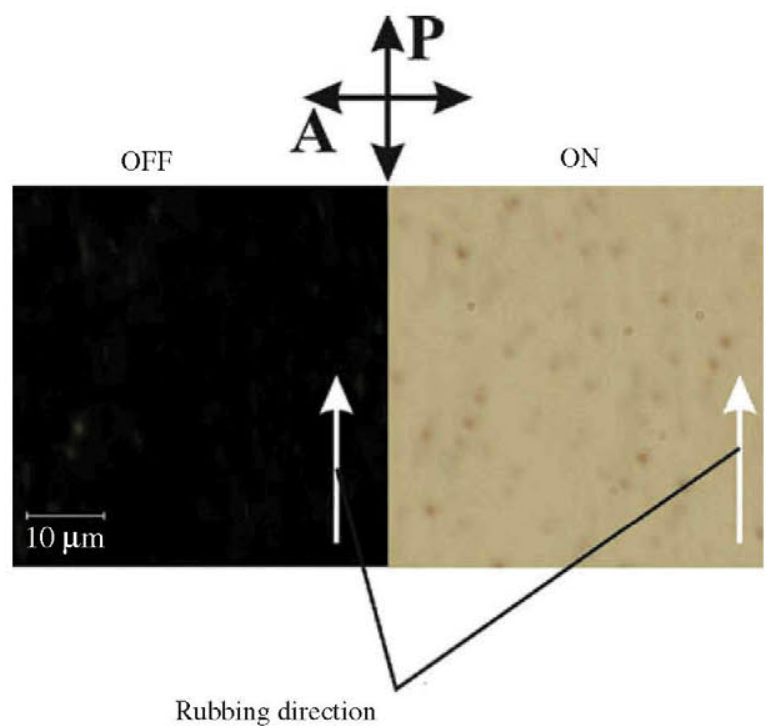

Figure 8. (colour online) Polarising microscopic textures of cell filled with W-304 in its OFF and ON states.

It can be noticed that the systems with the inversion of helical twist sense give the possibility of obtaining very long pitch in antiferroelectric phase. From obtained results, it can be conclude that the temperature of helical twist sense inversion can be easily controlled by proper adjustment of the mixture composition.

The antiferroelectric mixture (W-304) with longer pitch than W-1000 was obtained. It is orthoconic at the temperature below $60^{\circ} \mathrm{C}$, wherein the tilt angle is fixed and not changes with temperature. It has the same advantages as mixture W-1000 (i.e., high tilt angle and the full relaxation of an anticlinic state at the zero electric field). Moreover, the novelty of its electro-optical properties is the good symmetry response and lower switching voltage.

\section{Funding}

This work was supported by the NCN [under grant UMO2011/03/N/ST4/0136] and MNiSW [under grant PBS1/B3/9/ 2012].

\section{References}

[1] Lagerwall S, Dahlgren A, Jagemalm P, Rudquist P, D'Have K, Pauwels H, Dąbrowski R, Drzewiński W. Unique electro-optical properties of liquid crystals designed for molecular optics. Adv Funct Mater. 2001:11:87-94. doi:10.1002/1616-3028(200104)11:2\&1t; 87::AID-ADFM87\&gt;3.0.CO;2-E.

[2] Li J, Takezoe H, Fukuda A. Novel temperature dependences of helical pitch in ferroelectric and antiferroelectric chiral smectic liquid crystals. Jpn J Appl Phys. 1991;30:532-536. doi:10.1143/JJAP.30.532.

[3] Rudquist P. Orthoconic antiferroelectric liquid crystals. Liq Cryst. 2013;40:1678-1697. doi:10.1080/ 02678292.2013 .828331$.

[4] Quintana X, Gayo JL, Rodrigo C, Urruchi V, Otón JM. Addressing waveforms for tristable AFLCs in active matrix displays. Ferroelectrics. 2000;246:211218. doi: $10.1080 / 00150190008230068$.

[5] Tykarska M, Czerwiński M, Miszkurka J. Influence of temperature and terminal chain length on helical pitch in homologue series nH6Bi. Liq Cryst. 2010;37:487495. doi:10.1080/02678291003686880.

[6] Tykarska M, Czerwiński M, Żurowska M. The temperature and concentration dependence of helical pitch in the mixtures of antiferroelectric compounds with the opposite helical twist sense. Liq Cryst. 2011;38:561566. doi:10.1080/02678292.2011.558217.

[7] Czerwiński M, Tykarska M, Dąbrowski R, Chełstowska A, Żurowska M, Kowerdziej R, 
Jaroszewicz LR. The influence of structure and concentration of cyano-terminated and terphenyl dopants on helical pitch and helical twist sense in orthoconic antiferroelectric mixtures. Liq Cryst. 2012;39:14981502. doi: $10.1080 / 02678292.2012 .723047$.

[8] Sage I. Thermochromic liquid crystals. Liq Cryst. 2011;38:1551-1561. doi:10.1080/02678292.2011.631302.

[9] Piecek W, Perkowski P, Raszewski Z, Morawiak P, Żurowska M, Dąbrowski R, Czupryński K. Long pitch orthoconic antiferroelectric binary mixture for display applications. Mol Cryst Liq Cryst. 2010;525:140-152. doi:10.1080/15421401003796223.

[10] Piecek W, Bubnov A, Perkowski P, Morawiak P, Ogrodnik K, Rejmer W, Żurowska M, Hamplová $V$, Kašpar M. An effect of structurally noncompatible additive on the properties of a long-pitch orthoconic antiferroelectric mixture. Phase Transit. 2010;83:551-563. doi:10.1080/ 01411594.2010 .499496$.

[11] Żurowska M, Dąbrowski R, Dziaduszek J, Skrzypek K, Filipowicz M, Rejmer W, Czupryński K, Bennis N,
Otón JM. Influence of alkoxy chain length and fluorosubstitution on mesogenic and spectral properties of high tilted antiferroelectric esters. J Mater Chem. 2011;21:2144-2153. doi:10.1039/c0jm02015j.

[12] Dąbrowski R, Kula P, Raszewski Z, Piecek W, Otón JM, Spadło A. New orthoconic antiferroelectrics useful for applications. Ferroelectrics. 2010;395:116-132. doi:10.1080/00150191003684128.

[13] Raszewski Z, Kędzierski J, Perkowski P, Piecek W, Rutkowska J, Kłosowicz S, Zieliński J. Refractive indices of the MHPB(H)PBC and MHPB(F)PBC antiferroelectric liquid crystals. Ferroelectrics. 2002;276:289-300. doi:10.1080/00150190214411.

[14] Kuczyński W, Lagerwall ST, Matuszczyk M, Skarp K, Stebler B, Wahl J. Fast-switching low-temperature liquid crystal mixtures. Mol Cryst Liq Cryst. 1987;146:173-187. doi:10.1080/00268948708071812.

[15] Rodrigo C, Quentel S, Sabater J, Quintana X, Oton JM. Flickering and stability in AFLC analog grayscale. Ferroelectrics. 1996;178:55-63. doi:10.1080/ 00150199608008347. 